\title{
Fluoride toothpaste prevents caries
}

\author{
In children and adolescents, is brushing with fluoride toothpaste more \\ effective at reducing decay than brushing with a nonfluoride paste?
}

\author{
Marinho VCC, Higgins JPT, Sheiham A, Logan S. Fluoride \\ toothpastes for preventing dental caries in children and \\ adolescents (Cochrane Review). The Cochrane Library 2003; Issue \\ 1. Oxford: Update Software
}

Data sources The Cochrane Oral Health Group's Trials Register, the Cochrane Central Register of Controlled Trials, MEDLINE (1966-January 2000) and several other databases were sources. Journals and reference lists of articles were handsearched and selected authors and manufacturers were contacted.

Study selection Randomised controlled trials (RCT) or quasi-RCT that had blind outcome assessment, compared fluoride toothpaste with placebo in children up to 16 years of age over at least 1 year were included. The main outcome was caries increment measured by the change in decayed, missing and filled tooth surfaces (D(M)FS).

Data extraction and synthesis Inclusion decisions, quality assessment and data extraction were duplicated in a random sample of onethird of the studies, and consensus was achieved by discussion or by a third party. Authors were contacted for missing data. The primary measure of effect was the prevented fraction (PF), that is, the difference in caries increments between the treatment and control groups expressed as a percentage of the increment in the control group. Random-effects meta-analyses were performed where data could be pooled. Potential sources of heterogeneity were examined in randomeffects meta-regression analyses.

Results In total, 74 studies were included of which 70 contributed to the meta-analysis (42300 children). The pooled PF was 24\% (95\% confidence interval $(\mathrm{Cl}), 21-28 \%, P<0.0001)$. This means that 1.6 children need to brush with a fluoride rather than nonfluoride toothpaste over 3 years to prevent $1 \mathrm{D}(\mathrm{M}) \mathrm{FS}$ in populations with a caries increment of 2.6 D(M)FS per year (or 3.7 children in populations with a caries increment of $1.1 \mathrm{D}(\mathrm{M}) \mathrm{FS}$ per year). There was statistically significant heterogeneity within the studies. The effect of fluoride toothpaste increased with higher baseline levels of $D(M) F S$, higher fluoride concentration, higher frequency of use and supervised brushing, but was not influenced by exposure to water fluoridation. There is little information concerning deciduous dentition or adverse effects (fluorosis).

Conclusions Supported by more than half a century of research, the benefits of fluoride toothpastes are firmly established. Taken together, the trials are of relatively high quality, and provide clear evidence that fluoride toothpastes are efficacious in preventing caries.

\section{Commentary}

The objectives of the current review were to determine the effectiveness and safety of fluoride toothpastes in the prevention of caries in children, and to examine factors potentially modifying their effect. In contrast to many other Cochrane reviews, a considerable number of studies fulfilling the inclusion criteria were available for review. The pooled estimate for the observed

Address for correspondence: Emma Tavender, Review Group Co-ordinator, Cochrane Oral Health Group, University Dental Hospital of Manchester, Higher Cambridge Street, Manchester M15 6FH, UK. E-mail: emma.tavender@man.ac.uk percentage-difference in caries increment was $24 \%$ and its $95 \%$ CI was narrow enough to leave no doubt about the beneficial effect of brushing with fluoride toothpaste compared with brushing with a nonfluoride paste. The intention was to assess effectiveness. As a matter of fact, many of the studies included in the review were explanatory trials with the aim of finding out the effect of fluoridated toothpaste under ideal conditions (efficacy). Pragmatic trials with an explicit aim to assess the effect under the everyday conditions in real life (effectiveness) are regrettably still rare. With such a firm efficacy, however, one can safely assume that even the effectiveness is considerable. It is noteworthy that even children who had a low level of caries increment and who were exposed to fluoridated water or fluoride from other sources did benefit from using fluoridated toothpaste. At the moment the evidence on the beneficial effect of fluoride toothpastes is so strong that ethical reasons prevent further RCT in which brushing with a fluoride toothpaste is compared with brushing with a nonfluoride paste.

The clear and statistically significant heterogeneity of the studies calls for further examination of the role of factors that are modifying the effect of fluoridated toothpastes. For instance, the implication of fluoride concentration still remains somewhat unclear since an overwhelming majority of the studies have considered the effect of toothpastes containing $1000 \mathrm{ppm}$ fluoride against a nonfluoride paste. The fact that toothpaste containing $1000 \mathrm{ppm}$ fluoride has become a sort of golden standard does not imply that this concentration would be the ideal one. Among the reviewed studies, only a few had considered the effect of 1500 and $2500 \mathrm{ppm}$ pastes and none included any greater concentrations than those. Consequently we still do not know whether toothpastes with higher fluoride concentrations would be preferable and, if so, whether they should be used generally or only by individuals at high risk of caries. The same uncertainty holds for the low-fluoride toothpastes. For infants and toddlers we need to balance the risk of developing carious lesions with the risk of dental fluorosis, and more evidence is still required with regard to optimal fluoride concentrations for children of different ages and with different levels of exposure to fluoride from other sources. Even the effectmodification by the daily frequency of brushing with fluoridated toothpaste needs to be studied in more detail. We know for sure that two times a day is better than once, but the marginal utility of each of the additional daily brushing times is not known. There is still a need for good quality RCT examining different aspects of fluoridated toothpastes.

\section{Practice point}

- Fluoride toothpaste prevents caries and has a greater effect in people with more disease.

\section{Hannu Hausen}

Institute of Dentistry, University of Oulu, Finland

Evidence-Based Dentistry (2003) 4, 28.

doi:10.1038/sj.ebd.6400176 